\title{
BunNourology open Safety and efficacy of lacosamide versus phenytoin for refractory seizures in neurosurgical patients
}

\author{
Sarah Schaidle (D) , Kasey Greathouse, Deepika McConnell
}

To cite: Schaidle S, Greathouse $\mathrm{K}$, McConnell D. Safety and efficacy of lacosamide versus phenytoin for refractory seizures in neurosurgical patients. BMJ Neurology Open 2021;3:e000144. doi:10.1136/ bmjno-2021-000144

Received 24 February 2021 Accepted 06 June 2021

\section{Check for updates}

(c) Author(s) (or their employer(s)) 2021. Re-use permitted under CC BY-NC. No commercial re-use. See rights and permissions. Published by BMJ.

Department of Pharmacy, Northwestern Memorial Hospital Chicago, Illinois, USA

Correspondence to Dr Sarah Schaidle; sarah.schaidle@gmail.com

\section{ABSTRACT}

Background Postoperative neurosurgical patients have increased risk of seizures. Traditional anti-epileptics, such as phenytoin, are not always effective and cause adverse effects. Levetiracetam is the first-line therapy due to its similar efficacy and more favourable side effect profile. However, many patients continue to seize despite adequate dosing. Lacosamide has been used for refractory seizures and may offer similar seizure control without the negative aspects of traditional agents. The purpose of this study is to evaluate if lacosamide is as safe and effective as phenytoin in terminating seizures in neurosurgical patients already on levetiracetam.

Methods This retrospective, single-centre cohort study identified neurosurgical intensive care unit (ICU) patients $\geq 18$ years old who had received levetiracetam plus either phenytoin or lacosamide at Northwestern Memorial Hospital between 1 January 2016 and 31 August 2017. The primary endpoint was treatment failure and the secondary endpoint was safety assessed by liver function tests, blood pressure, heart rate and ECG.

Results 70 patients were included in this study, 52 in the phenytoin group and 18 in the lacosamide group. Both phenytoin and lacosamide groups had similar treatment failure rates ( $25 \%$ vs $22 \%$ respectively, $p=1)$. Phenytoin use resulted in a mean decrease in systolic blood pressure of $20.9 \mathrm{~mm} \mathrm{Hg}$ compared with $9.8 \mathrm{~mm} \mathrm{Hg}$ in the lacosamide group $(p=0.019)$. There were no statistically significant differences in the rates of other adverse effects. Conclusions The use of lacosamide for refractory seizures in neurosurgical ICU patients was associated with similar failure rates, but fewer adverse effects when compared with phenytoin.

\section{INTRODUCTION}

Postoperative neurosurgical patients have increased risk of seizures, particularly after subdural haematoma evacuation or tumour resection, with the highest risk occurring early in the postoperative setting. ${ }^{1}$ Seizures contribute substantially to increased morbidity and mortality due to elevated intracranial pressure and significant increases in systemic oxygen consumption. Furthermore, the incidence and duration of seizures directly correlates with morbidity and mortality in these patients. ${ }^{2}$ Therefore, intravenous medications are preferred in the treatment of acute seizures for more rapid response. Traditionally, fosphenytoin or phenytoin, valproic acid and phenobarbital have been used most often for seizure cessation. However, these older anti-epileptic drugs (AED) are not always effective and are associated with adverse effects and drug interactions. A newer AED, levetiracetam, has become the first-line therapy due to its similar efficacy alongside a more favourable side effect profile, less frequent therapeutic drug monitoring and minimal drug interactions. Furthermore, levetiracetam has been the preferred first-line AED at Northwestern Memorial Hospital (NMH) for neurosurgical patients with brain tumours to avoid drug interactions with chemotherapy regimens. Unfortunately, many patients continue to seize despite adequate dosing of levetiracetam, necessitating a second AED. In the past, phenytoin had been the most common second-line agent used at this institution, but recently lacosamide has become more widely used for this indication.

Lacosamide was approved by the Food and Drug Administration in 2009 for adjunct and monotherapy for partial seizures. It works by controlling neuronal hyperexcitability via selective sodium channel slow inactivation and binding to collapsin response mediator protein-2. ${ }^{3}$ Compared with phenytoin, it does not require therapeutic drug monitoring, has limited adverse effects and drug interactions and is generally well tolerated. Our study aimed to evaluate the efficacy and safety of lacosamide versus phenytoin when added to levetiracetam in neurosurgical patients with refractory seizures.

\section{METHODS}

This retrospective cohort study was conducted using the electronic health record to identify neurosurgical intensive care unit (ICU) patients 18 years of age or older who have received levetiracetam plus either phenytoin 
or lacosamide at NMH between 1 January 2016 and 31 August 2017. Manual chart review was then conducted to evaluate inclusion and exclusion criteria. Pregnant patients and patients presenting in status epilepticus were excluded.

The primary endpoint for this study was treatment failure defined as the addition of a third anti-epileptic medication or recurrent seizure as evident by continuous electroencephalogram (EEG) monitoring. The comprehensive list of anti-epileptic medications that could be considered as a third-line agent included: phenytoin, fosphenytoin, lacosamide, valproic acid, propofol, phenobarbital, pentobarbital, carbamazepine, topiramate, lorazepam, midazolam, clonazepam, diazepam and clobazam. The secondary endpoint was safety assessed by liver function tests (LFT), blood pressure, heart rate and ECG changes with the administration of each drug. Elevation in LFT was defined as a twofold increase in aspartate transaminase or alanine transaminase during the current hospital admission after the administration of the AED. Hypotension was defined as a decrease in systolic blood pressure (SBP) $\geq 20 \mathrm{~mm} \mathrm{Hg}$ within 1 hour of AED administration compared with baseline. Bradycardia was defined as heart rate $<60 \mathrm{bpm}$ within 1 hour of AED administration compared with baseline. PR interval prolongation was defined as PR interval $>200 \mathrm{~ms}$ during current hospital admission after the administration of the AED. Per hospital policy, the maximum infusion rate of phenytoin was $25 \mathrm{mg} / \mathrm{min}$.

Descriptive statistics were calculated, including frequencies and proportions for categorical data, and means for continuous data. A $\chi^{2}$ test was used for categorical variables and student's individual t-test was used for continuous data analyses. P value of $<0.05$ was considered statistically significant. Analyses were completed using IBM SPSS Statistics V.23.

\section{RESULTS}

A total of 156 adult neurosurgical ICU patients were identified for inclusion. Eighty-six of these patients were excluded due to the study medications being home medications, ordered for another indication, or ordered but never administered to the patient. Additionally, five of these patients presented in status epilepticus. After exclusions, 70 patients, 52 in the phenytoin group and 18 in the lacosamide group, were included in the analysis (figure 1). Baseline characteristics were similar in both cohorts (table 1 ). The median age was 55 years old and $39 \%$ were women. The most common admission diagnosis was brain tumour followed by subdural haematoma. Thirty-seven percent of patients in the phenytoin group and $39 \%$ of patients in the lacosamide group had a history of seizures. Most patients (93\%) underwent a craniotomy. Eighty-one percent of patients were monitored by continuous EEG during hospital admission. The decision to give either phenytoin or lacosamide as the second-line agent was based on provider preference. The loading

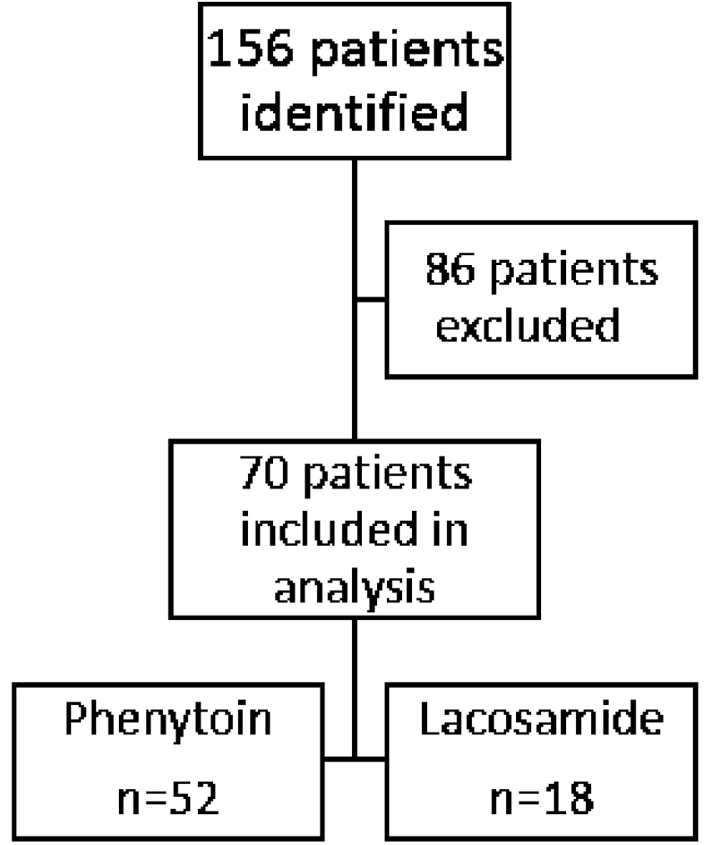

Figure 1 One hundred and fifty-six patients were identified for study inclusion. Of these patients, 86 were excluded.

Patients were excluded if medications of interest were home medications $(n=56)$, ordered but never given $(n=22)$ or used for another indication $(n=3)$. Patients who presented in status epilepticus were also excluded $(n=5)$. This left 52 patients in the phenytoin group and 18 patients in the lacosamide group for analysis.

dose of phenytoin ranged from 10 to $24 \mathrm{mg} / \mathrm{kg}$ (500 to $2000 \mathrm{mg}$ ) with an average loading dose of $16.9 \pm 2.8 \mathrm{mg}$ / $\mathrm{kg}(1333 \pm 323 \mathrm{mg})$ based on actual body weight. Free phenytoin levels were collected on all patients in the phenytoin cohort and ranged from 0.42 to $3.15 \mathrm{mcg} / \mathrm{mL}$. Sixty-nine percent of free phenytoin levels were within goal range of $1-2 \mathrm{mcg} / \mathrm{mL}$ with $29 \%$ above goal range and only $2 \%$ below goal range. The lacosamide loading dose was $200 \mathrm{mg}$ for all patients followed by $100 \mathrm{mg}$ two times per day for maintenance dosing.

Thirteen patients (25\%) failed therapy with phenytoin while four patients $(22 \%)$ failed therapy with lacosamide $(\mathrm{p}=1)$ (table 2). Treatment failure was determined by recurrent seizures captured by continuous EEG monitoring in 12 patients (92\%) in the phenytoin group and 3 patients $(75 \%)$ in the lacosamide group. Lacosamide was added as the third AED in $84.6 \%$ of patients who failed phenytoin. For those patients who failed treatment with lacosamide, phenytoin was added as the third AED 50\% of the time and valproic acid was used the other $50 \%$ of the time. Benzodiazepines were ultimately used for seizure control in $21 \%$ of patients in the phenytoin group and $11 \%$ in the lacosamide group. Of the benzodiazepines, lorazepam was used most often (69\%). Hypotension occurred in $55 \%$ of patients in the phenytoin group and $36 \%$ of patients in the lacosamide group ( $\mathrm{p}=0.198)$ (table 3). The mean drop in SBP was $20.9 \mathrm{~mm} \mathrm{Hg}$ in the phenytoin group and $9.8 \mathrm{~mm} \mathrm{Hg}$ in the lacosamide 
Table 1 Baseline characteristics of the patients, according to treatment group.

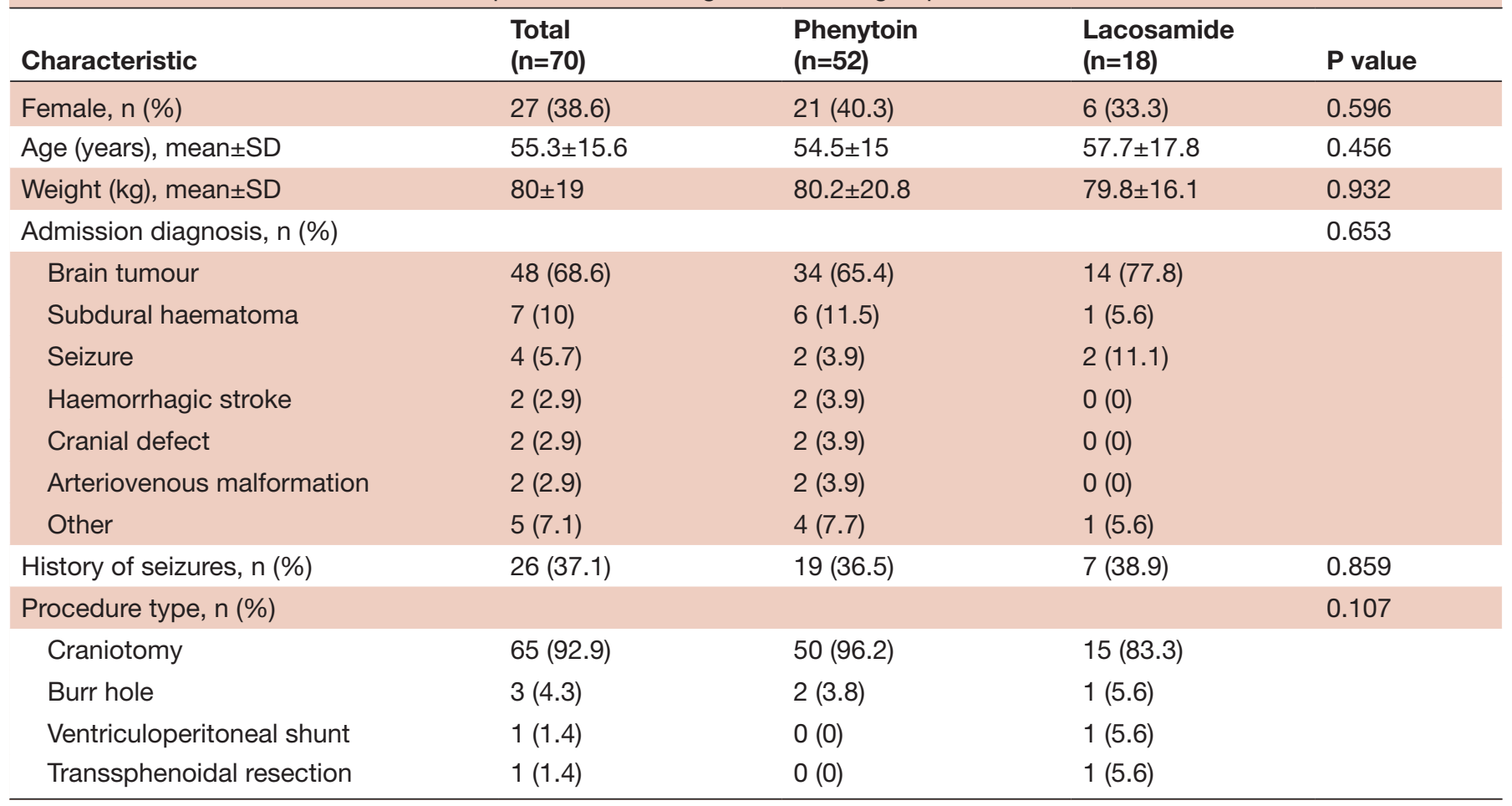

group, which was statistically significant $(p=0.019)$. Six patients in the phenytoin group had clinically significant hypotension ( $\mathrm{SBP}<80 \mathrm{~mm} \mathrm{Hg}$ ) with four of these patients requiring vasopressor therapy immediately after the administration of phenytoin. In the phenytoin group, bradycardia and LFT elevation occurred in $9.8 \%$ and $29 \%$ of patients, respectively. There were no incidences of bradycardia or LFT elevation in the lacosamide group. Out of the 46 patients with ECG completed in the phenytoin group, there were no incidences of PR interval prolongation. However, 1 patient out of the 15 patients $(6.7 \%)$ with ECG completed in the lacosamide group experienced PR prolongation.

Table 2 Incidence of primary outcome and additional antiepileptic drugs used.

\begin{tabular}{|c|c|c|c|}
\hline Outcome & $\begin{array}{l}\text { Phenytoin, } \\
\mathrm{n}=52\end{array}$ & $\begin{array}{l}\text { Lacosamide, } \\
\mathrm{n}=18\end{array}$ & P value \\
\hline $\begin{array}{l}\text { Treatment failure, } \\
\mathrm{n}(\%)\end{array}$ & $13(25)$ & $4(22.2)$ & 1 \\
\hline \multicolumn{4}{|c|}{ Third anti-epileptic drug added, n (\%) } \\
\hline Phenytoin & $0(0)$ & $2(50)$ & \\
\hline Lacosamide & $11(84.6)$ & $0(0)$ & \\
\hline Propofol & $2(15.4)$ & $0(0)$ & \\
\hline Valproic acid & $0(0)$ & $2(50)$ & \\
\hline $\begin{array}{l}\text { Benzodiazepine } \\
\text { use, n (\%) }\end{array}$ & $11(21.2)$ & $2(11)$ & \\
\hline Lorazepam & 7 (63.6) & $2(100)$ & \\
\hline Midazolam & $4(36.4)$ & $0(0)$ & \\
\hline
\end{tabular}

\section{DISCUSSION}

This study found similar efficacy between lacosamide and phenytoin for control of refractory seizures in neurosurgical ICU patients which could potentially be explained by their similar mechanisms of action through voltagegated sodium channels. Resistant-to-treatment seizures are common in postoperative neurosurgical ICU patients and there are no clear guidelines on the appropriate AED regimen to use for this patient population. ${ }^{1}$ Phenytoin has traditionally been used in these patients as it has been wellstudied and is familiar to prescribers. However, it requires therapeutic drug monitoring and has multiple adverse effects and drug interactions. Lacosamide is a newer AED that has a more favourable pharmacokinetic profile with

Table 3 Safety outcomes based on treatment group.

\begin{tabular}{|c|c|c|c|}
\hline Outcome & $\begin{array}{l}\text { Phenytoin, } \\
n=52\end{array}$ & $\begin{array}{l}\text { Lacosamide, } \\
n=18\end{array}$ & $P$ value \\
\hline Hypotension, n (\%)* & $26(55.3)$ & $5(35.7)$ & 0.198 \\
\hline $\begin{array}{l}\text { Mean decrease } \\
\text { in systolic blood } \\
\text { pressure } \pm S D, \mathrm{~mm} \mathrm{Hg}\end{array}$ & $20.9 \pm 18.5$ & $9.8 \pm 11.5$ & 0.019 \\
\hline LFT elevation, n (\%)† & $5(29.4)$ & $0(0)$ & 0.539 \\
\hline $\begin{array}{l}\text { PR interval } \\
\text { prolongation, } n(\%) \ddagger\end{array}$ & $0(0)$ & $1(6.7)$ & 0.246 \\
\hline Bradycardia, n (\%)§ & $5(9.8)$ & $0(0)$ & 0.316 \\
\hline
\end{tabular}

${ }^{*} n=47$ and $n=14$, respectively. $\dagger n=17$ and $n=3$, respectively. $\neq n=46$ and $n=15$, respectively. $\S n=51$ and $n=18$, respectively. 
minimal adverse effects and drug interactions. ${ }^{4}$ This study showed that the use of lacosamide for refractory seizures in neurosurgical ICU patients was associated with similar failure rates when compared with phenytoin.

Of the 17 patients who failed therapy, $88 \%$ were deemed as treatment failure due a recurrent seizure noted on continuous EEG monitoring which is a more accurate marker of breakthrough seizures than the addition of a third AED. Furthermore, phenytoin failure could potentially be due to suboptimal dosing resulting in subtherapeutic levels in a critically ill population, however this study had $69 \%$ of free phenytoin levels within goal range of 1-2 $\mathrm{mcg} / \mathrm{mL}$. Additionally, phenytoin was associated with higher rates of adverse effects including hypotension, bradycardia and LFT elevation which may partially be attributed to the supratherapeutic free phenytoin levels found in $29 \%$ of patients in the phenytoin cohort.

Several small studies have evaluated the general use of lacosamide in patients with brain tumour-related epilepsy (BTRE) and found it to be effective and well tolerated. The NEOPLASM study was a multicentre, retrospective, observational study that evaluated the efficacy and tolerability of lacosamide in 105 adult patients with BTRE over 6 months. ${ }^{5}$ Thirty-one percent of patients were seizurefree and $66 \%$ were responders, defined as a $\geq 50 \%$ seizure reduction at 6 months after the initiation of lacosamide. This study also found that the rates of seizure freedom and response rates in the overall population compared with those who switched to lacosamide because of a lack of efficacy with their previous AED were similar. Lastly, this study noted that the number of patients with adverse effects was lower in those receiving levetiracetam plus lacosamide than those receiving other dual therapy combinations, although this difference was not statistically significant. Adverse effects associated with lacosamide use occurred in $42 \%$ of patients with the most common being somnolence, fatigue and dizziness. Only $4.7 \%$ of these adverse effects led to the discontinuation of lacosamide. Saria et al completed a retrospective chart review of 70 brain tumour patients who had received lacosamide for seizure management to determine the tolerability and activity of lacosamide in patients with brain tumours. ${ }^{3}$ In this study, $66 \%$ of patients reported a decrease in seizures and $83 \%$ of these patients reported $\geq 50 \%$ decrease in seizure frequency. Seventy-seven percent of patients did not require additional AEDs following lacosamide therapy. Twenty-three percent of patients reported adverse effects with the most common being fatigue, dizziness, nausea, confusion and weakness. Finally, a case series of 14 patients suffering from BTRE evaluated the efficacy and tolerability of lacosamide as an add-on therapy. ${ }^{6}$ The mean duration of follow-up was 5.4 months and at last follow-up, the mean seizure number was reduced from 15.4 to 1.9 per month $(\mathrm{p}<0.022)$ after initiation of lacosamide. Forty-three percent of these patients were seizurefree and only one patient had to discontinue lacosamide due to side effects including dizziness and blurred vision. In addition to these previous studies, the results of the present study support the use of lacosamide for refractory seizures in neurosurgical ICU patients.

Limitations of this study include the retrospective and single-centre design. Due to its retrospective nature, the timing of medication administration was dependent on the time the medication was charted in the medication administration record. The incidence and timing of seizures was based on documentation in neurosurgical notes if the patient was not on continuous EEG monitoring. Furthermore, it was also only possible to assess the secondary outcome of safety on patients who had the laboratory values and vitals documented. We were unable to control for certain factors that may have influenced the rate of adverse effects in our patient population including concomitant medications and comorbidities. Lastly, due to the small sample size, this study was not powered to detect a difference in treatment failure rates and adverse effects associated with each medication.

\section{CONCLUSIONS}

In conclusion, lacosamide was associated with similar failure rates, but fewer adverse effects when compared with phenytoin for refractory seizures in neurosurgical ICU patients.

Contributors KG and DM conceived and designed the study idea. SS completed data collection and analysis of the results. SS wrote the manuscript with input from all authors. SS, KG and DM are all guarantors of the study.

Funding The authors have not declared a specific grant for this research from any funding agency in the public, commercial or not-for-profit sectors.

Competing interests None.

Patient consent for publication Not required.

Ethics approval This study was approved under the Northwestern University Institutional Review Board.

Provenance and peer review Not commissioned; externally peer reviewed. Data availability statement Data are available upon reasonable request.

Open access This is an open access article distributed in accordance with the Creative Commons Attribution Non Commercial (CC BY-NC 4.0) license, which permits others to distribute, remix, adapt, build upon this work non-commercially, and license their derivative works on different terms, provided the original work is properly cited, appropriate credit is given, any changes made indicated, and the use is non-commercial. See: http://creativecommons.org/licenses/by-nc/4.0/.

ORCID iD

Sarah Schaidle http://orcid.org/0000-0001-5074-839X

\section{REFERENCES}

1 Varelas PN, Spanaki MV, Mirski MA. Seizures and the neurosurgical intensive care unit. Neurosurg Clin N Am 2013;24:393-406.

2 Cherry S, Judd L, Muniz JC, et al. Safety and efficacy of lacosamide in the intensive care unit. Neurocrit Care 2012;16:294-8.

3 Saria MG, Corle C, Hu J, et al. Retrospective analysis of the tolerability and activity of lacosamide in patients with brain tumors: clinical article. J Neurosurg 2013;118:1183-7.

4 UCB, Inc. Vimpat® [package insert. Smyrna, GA: UCB, Inc, 2009.

5 Villanueva V, Saiz-Diaz R, Toledo M, et al. Neoplasm study: real-life use of lacosamide in patients with brain tumor-related epilepsy. Epilepsy Behav 2016;65:25-32.

6 Maschio M, Dinapoli L, Mingoia M, et al. Lacosamide as add-on in brain tumor-related epilepsy: preliminary report on efficacy and tolerability. J Neurol 2011;258:2100-4. 